\title{
Polarized fluorescence measurements of orientational order in a uniaxial liquid crystal
}

\section{Chapoy, L. Lawrence; DuPré, Donald B.}

Published in:

Journal of Chemical Physics

Link to article, DOI:

$10.1063 / 1.437717$

Publication date:

1979

Document Version

Publisher's PDF, also known as Version of record

Link back to DTU Orbit

Citation (APA):

Chapoy, L. L., \& DuPré, D. B. (1979). Polarized fluorescence measurements of orientational order in a uniaxial liquid crystal. Journal of Chemical Physics, 70(5), 2550-2553. https://doi.org/10.1063/1.437717

\section{General rights}

Copyright and moral rights for the publications made accessible in the public portal are retained by the authors and/or other copyright owners and it is a condition of accessing publications that users recognise and abide by the legal requirements associated with these rights.

- Users may download and print one copy of any publication from the public portal for the purpose of private study or research.

- You may not further distribute the material or use it for any profit-making activity or commercial gain

- You may freely distribute the URL identifying the publication in the public portal

If you believe that this document breaches copyright please contact us providing details, and we will remove access to the work immediately and investigate your claim. 


\title{
Polarized fluorescence measurements of orientational order in a uniaxial liquid crystal
}

\author{
L. Lawrence Chapoy and Donald B. DuPréa) \\ Instituttet for Kemiindustri, The Technical University of Denmark, 2800 Lyngby, Denmark \\ (Received 10 March 1978) \\ The second and fourth orientational order parameters, $\left\langle P_{2}\right\rangle$ and $\left\langle P_{4}\right\rangle$, have been measured throughout \\ the liquid crystalline phase of $p$-methoxybenzylidene- $p^{\prime}-n$-butylaniline (MBBA) using small quantities of \\ a fluorescent probe. Complications of rotational Brownian motion and the intramolecular transfer of \\ excitation energy were considered in the analysis. The results are in agreement with previous Raman \\ measurements on the doped liquid crystal.
}

An unambiguous formulation of the long range orientational distribution function, $f(\theta)$, in uniaxial liquid crystals is obtained by writing the function as a truncated series of the even Legendre polynomials ${ }^{1}: f(\theta)$ $=\Sigma c_{l} P_{l}(\cos \theta)$, where $P_{0}=1, P_{2}=\frac{1}{2}\left(3 \cos ^{2} \theta-1\right), P_{4}$ $=\frac{1}{8}\left(35 \cos ^{4} \theta-30 \cos ^{2} \theta+3\right), \cdots$ and the coefficients $c_{l}$ $=(2 l+1) / 2\left\langle P_{t}(\cos \theta)\right\rangle$ are experimentally determinable moments of the distribution. X-ray; optical birefringence; ir, visible, and uv dichroism measurements; and magnetic resonance techniques are the most frequently cited means of determining the degree of liquid crystalline order through the parameter $S=\left\langle P_{2}\right\rangle$, the second moment of the distribution. Both the second and fourth moments are available, however, from suitable analysis of resonance linewidths of electron spin probes ${ }^{2}$ and of polarized $\operatorname{Raman}^{3-6}$ and fluorescent emission spectra $^{3,4}$ of active sites, usually in a doped liquid crys tal. The fluorescence technique has many things in common with the Raman method in that it is also a two step spectroscopic process modulated by molecular motion. The Raman effect is, however, effectively instantaneous $\left(\sim 10^{-12} \mathrm{sec}\right)$ whe reas fluorescent emission may follow absorptive excitation by many nanoseconds, perhaps even emanating polarized along a different oscillator axis in the molecule. The latter factors lead to experimental and interpretative difficulties particularly when rotational diffusion processes occur. There also exist complications when intermolecular energy transfer occurs which depolarizes emission in an unpredictable manner.

We have recently considered ${ }^{7}$ the combined effects of rotational Brownian motion of a fluorescent site during the lifetime of its excitation and the inherent nonparallelism of absorption and emission oscillators. An expression was derived for the anisotropy of emission, $r$, which contains the possibility of a nonrandom distribution of fluorescent sites before the photo-selection process, such as is present in liquid crystals. In general,

$r\left(\tau / \tau_{R} ; \delta\right)=\frac{1}{2} \cdot\left(\frac{3 \overline{\cos ^{4} \theta}-\overline{\cos ^{2} \theta}}{\overline{\cos ^{2} \theta}}\right) \cdot\left(\tau / \tau_{R}+1\right)^{-1} \cdot P_{2}(\cos \delta)$,

where $\delta$ is the intramolecular energy transfer angle of the fluorophore and $\tau / \tau_{R}$ is the ratio of the fluorescence lifetime to the characteristic rotational relaxational

\footnotetext{
a) On leave from Department of Chemistry, University of
} Louisville, Louisville, Kentucky 40208. time of the probe in the host medium. (See Appendix.) In the limit of totally random molecular order, $\overline{\cos ^{2} \theta}=\frac{1}{3}$, $\overline{\cos ^{4} \theta}=\frac{1}{5}$ and Eq. (1) reduces to Perrin's equation ${ }^{8}$ :

$$
r=\frac{2}{5} \cdot P_{2}(\cos \delta) \cdot\left(\tau / \tau_{R}+1\right)^{-1} \text {. }
$$

It was shown that considerable misinterpretation of experimental data, and hence the form of the orientational distribution function, can occur if the analysis of the fluorescence experiment is not properly placed in the regime of $\delta$ and $\tau / \tau_{R}$. In this note we report the temperature dependence of the $\left\langle P_{2}\right\rangle,\left\langle P_{4}\right\rangle$ order parameters obtained from the polarized emission of small quantities of 4-dimethylamino-4' -nitrostilbene (DS) embedded in the liquid crystal $p$-methoxybenzylidene- $p^{\prime}-n$ butylaniline (MBBA).

The probe DS was selected for study as it has been previously shown ${ }^{9}$ to be soluble in MBBA with an absorption band shifted to the red of the liquid crystal primary uv-visible absorption. The molecule also shows no tendency to bleach when exposed to uv radiation over an extended period of time.

The intramolecular energy transfer vector $\delta$ and information about the rotational mobility of a probe mole cule can be obtained from measurements of $r$ as a function of temperature in viscous isotropic solvents. An intrinsic polarization anisotropy factor $r_{0}=\frac{2}{5} \cdot P_{2}(\cos \delta)$ internal to the fluorescent probe molecule can be measured utilizing Perrin's relation, Eq. (2), from the limit of $r$ as $T / \eta \rightarrow 0$ since $\tau / \tau_{R} \sim \tau T / \eta V$ where $T$ and $\eta$ are the temperature and solvent viscosity, respectively, and $V$ is the effective volume of the probe molecule. The value of $r_{0}$ so determined for DS in dimethylphthalate was close to the theoretical value for an ideal probe molecule with $P_{2}(\cos \delta)=0.91 \pm 0.02$. Figure 1 shows that $\tau / \tau_{R} \ll 1$ even at room temperature for this molecule and the complications of rotational diffusion can thus be neglected in this analysis. Eq. (1), simplifies to:

$$
r=\frac{9 \overline{\cos ^{4} \theta}-\overline{3 \cos ^{2} \theta}}{6 \overline{\cos ^{2} \theta}} \cdot P_{2}(\cos \delta)
$$

in this limit where the distribution of molecular orientations is static, though nonrandom.

In order to extracate $\overline{\cos ^{2} \theta}$ from $\overline{\cos ^{4} \theta}$ in Eq. (3) another experiment is necessary. A measurement of the absorption dichroic ratio, $D$, would be sufficient as: 


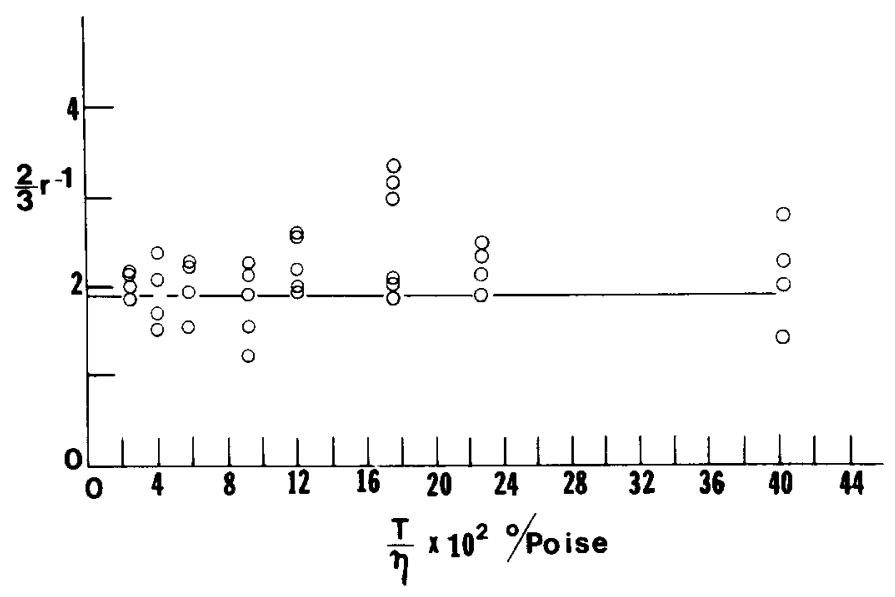

FIG. 1. Plot of $\frac{2}{3} r^{-1}$ vs $T / \eta$ for dimethylaminonitrostilbene (DS), $10^{-5} \mathrm{M}$, in the solvent dimethylphthalate.

$\overline{\cos ^{2} \theta}=D / D+2$. Choosing another fluorescence geometry will also provide a second expression for the moments which can be solved simultaneously with Eq. (3). If we define a new polarization anisotropy $\boldsymbol{r}^{\prime}$, measured in the right angle geometry with the incident polarization lying now in a plane normal to the major symmetry axis of the sample, then ${ }^{7}$ :

$$
\begin{aligned}
r^{\prime}= & \frac{\left(6 \overline{\cos ^{2} \theta}-5 \overline{\cos ^{4} \theta}-1\right)}{\left(5-6 \overline{\cos ^{2} \theta}+\overline{\cos ^{4} \theta}\right)+\left(6 \overline{\cos ^{2} \theta}-3 \overline{\cos ^{4} \theta}-3\right) \cos ^{2} \delta} \\
& \cdot P_{2}(\cos \delta) .
\end{aligned}
$$

In this experiment, the liquid crystal was doped at a concentration of $10^{-2} \mathrm{M}$ DS. Differential thermal analysis showed the clearing temperature depressed to $T_{c}$ $\approx 38^{\circ} \mathrm{C}$. Monodomain samples were prepared on glass slides by the familiar rubbing technique. The sample cell consisted of a thin film of the liquid crystal sandwiched in between two glass slides in direct contact with a common director axis. Absorption and fluorescent emission intensities were measured with polars parallel and perpendicular to the axis of the director. In the fluorescence experiments, the excitation polarization was also placed parallel and perpendicular to the director in order to obtain both $r$ and $r^{\prime}$. The instrumentation consisted of a Beckman DK-2A visible-ultraviolet double beam absorption spectrophotometer and a Perkin-Elmer MPF-2A fluorescence spectrophotometer. The latter is equipped with dual slits and monochromators. Excitation was performed at $445 \mathrm{~nm}$ where MBBA has no appreciable absorption and emission was observed at $570 \mathrm{~nm}$ with slits set at $10 \mathrm{~nm}$. In spite of extensive light scattering from the liquid crystal, complete resolution was attained between the broad scattering peak and the probe emission. The emission was a broad featureless structure centered at $570 \mathrm{~nm}$. The emission intensities were obtained in the "ratio mode" which compensates for fluctuations in excitation lamp intensity, thus stabilizing the observed signal. Additional stabilization of the signal is effected by averaging the signal in the real time domain using a Solartron 1860 Computing Digit.1 Voltmeter. This procedure averages out low frequency noise, which cannot be effectively removed by capacitative filtering. Precision in the determination of emission intensities is essential as the determination of $r$ involves small differences in large numbers. Dichroic ratios of the preparation were determined as a function of temperature throughout the liquid crystal region and the order parameter $S=\left\langle P_{2}\right\rangle$ calculated.

The determination of $\overline{\cos ^{4} \theta}$, and hence $\left\langle P_{4}\right\rangle$ proceeded as outlined above. The value of $r$ obtained in the isotropic phase of the liquid crystal mixture is approximately the same as that found in viscous liquids in the limit of $T / \eta-0\left[i_{\circ}\right.$ e. $\left.\frac{2}{5} \cdot P_{2}(\cos \delta)\right]$, further demonstrating that Brownian motion is not a problem in this probeliquid crystal system. Values of $\overline{\cos ^{2} \theta}$ and $\overline{\cos ^{4} \theta}$ thus obtained by both procedures are presented in Table I and are plotted in terms of $\left\langle P_{2}\right\rangle,\left\langle P_{4}\right\rangle$ in Fig. 2.

Analysis of random error leads to an estimated error $(\Delta)$ in the primary data variables: $\Delta D=0.30$ and $\Delta r$, $\Delta r^{\prime}=0.001$, respectively. Sources of systematic error include: error in anchoring of the liquid crystal to the sample container, misalignment of the preferred di-

TABLE I. Fluorescent emission anisotropies and moments of the orientational distribution

\begin{tabular}{|c|c|c|c|c|c|c|c|c|c|c|}
\hline $\begin{array}{l}T_{c}-T \\
\left({ }^{\circ} \mathrm{C}\right) \\
\end{array}$ & $r$ & $\cos ^{2} \theta^{2}$ & $\overline{\cos ^{4} \theta}$ & $\left\langle\boldsymbol{P}_{2}\right\rangle^{\mathbf{a}}$ & $\left\langle P_{4}\right\rangle$ & $r^{\prime}$ & $\overline{\cos ^{2} \theta}$ & $\cos ^{4} \theta$ & $\left\langle P_{2}\right\rangle$ & $\left\langle P_{4}\right\rangle$ \\
\hline 17.5 & 0.57 & 0.73 & 0.56 & 0.59 & 0.08 & 0.41 & 0.68 & 0.52 & 0.52 & 0.09 \\
\hline 15.5 & 0.56 & 0.73 & 0.56 & 0.59 & 0.08 & 0.40 & 0.66 & 0.50 & 0.49 & 0.10 \\
\hline 13.5 & 0.55 & 0.71 & 0.53 & 0.57 & 0.04 & 0.39 & 0.66 & 0.49 & 0.49 & 0.068 \\
\hline 11.5 & 0.54 & 0.70 & 0.52 & 0.55 & 0.02 & 0.38 & 0.64 & 0.48 & 0.47 & 0.050 \\
\hline 9.8 & 0.53 & 0.69 & 0.51 & 0.54 & 0.006 & 0.37 & 0.63 & 0.46 & 0.45 & 0.025 \\
\hline 8.0 & 0.52 & 0.67 & 0.49 & 0.51 & -0.01 & 0.36 & 0.62 & 0.45 & 0.43 & 0.014 \\
\hline 5.9 & 0.51 & 0.66 & 0.47 & 0.49 & -0.03 & 0.34 & 0.61 & 0.44 & 0.42 & -0.008 \\
\hline 3.7 & 0.49 & 0.63 & 0.44 & 0.45 & -0.06 & 0.33 & 0.59 & 0.42 & 0.38 & -0.015 \\
\hline 2.2 & 0.48 & 0.61 & 0.43 & 0.42 & -0.05 & 0.31 & 0.57 & 0.40 & 0.36 & -0.030 \\
\hline 0.9 & 0.47 & 0.59 & 0.40 & 0.38 & -0.07 & 0.26 & 0.54 & 0.37 & 0.32 & -0.035 \\
\hline $\begin{array}{l}\text { iso- } \\
\text { trope }\end{array}$ & 0.34 & 0.33 & 0.20 & 0.00 & 0.00 & $(0.00)$ & 0.33 & 0.20 & 0.00 & 0.00 \\
\hline
\end{tabular}
function for the liquid crystal MBBA.

${ }^{2}$ From optical dichroism. 


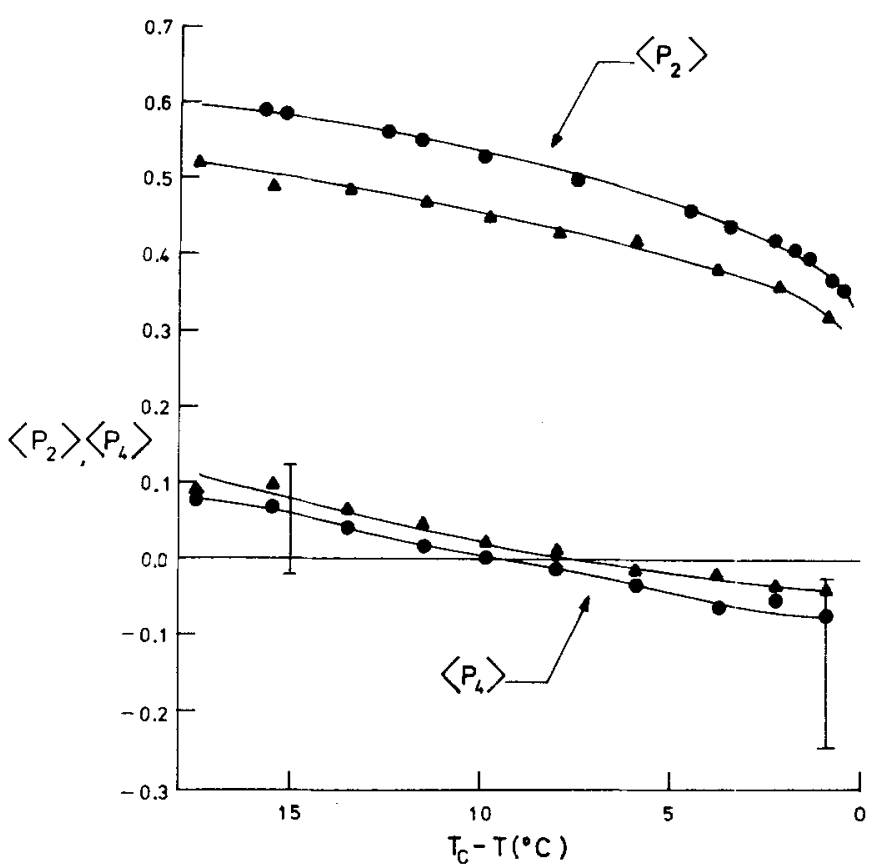

FIG. 2. Experimental values of the liquid crystal order parameters $\left\langle P_{2}\right\rangle$ and $\left\langle P_{4}\right\rangle$. [Triangles $(\Delta)$, from simultaneous solution of Eqs. (3) and (4); circles (0), from combination of fluorescent emission and optical dichroism data]. The error bars are those of an independent study of $\left\langle P_{4}\right\rangle$ by Raman scattering ${ }^{6}$ on a similarly doped sample of MBBA.

rector axis of the liquid crystal cell with respect to the optical axis of the spectrometer, internal misalignment of the optics of the spectrometer, polarization bias of the spectrometer, and interference due to light scattering. Polarization bias was corrected for by multiplying measured fluorescent intensities by a correction factor obtained from the isotropic phase where $\boldsymbol{r}^{\prime}$ should be identically zero. Light scattering from the liquid crystal medium was minimized by using a very thin specimen as described above. The probe molecule emission band is well separated and completely resolved from the scattering peak. The absorption band of the probe molecule is also well separated from the absorption band of MBBA. Our estimate of systematic error from other uncorrectable sources, obtained from measurements of a number of different sample preparations placed in the spectrometer compartments, leads to $\Delta D$ $=0.40$ and $\Delta r, r^{\prime}=0.02$, respectively. This brings the error bars of our measurements of $\left\langle P_{2}\right\rangle$ and $\left\langle P_{1}\right\rangle$ at $T-T_{c}=10^{\circ} \mathrm{C}$, for example, by the first procedure to $\Delta\left\langle P_{2}\right\rangle \sim 0.03,\left\langle P_{4}\right\rangle \sim 0.13$; and by the second procedure to $\Delta\left\langle P_{2}\right\rangle \sim 0.02, \Delta\left\langle P_{4}\right\rangle \sim 0.07$.

The $\left\langle P_{2}\right\rangle$ values from optical dichroism measurements are in good agreement with those obtained for MBBA by NMR, Raman, magnetic susceptibility, and optical methods. ${ }^{6}\left\langle P_{2}\right\rangle$ values obtained from the simultaneous solution of fluorescence depolarization Eqs. (3) and (4) are slightly and consistently lower than most other literature values, presumably due to differing contributions of systematic errors in the two procedures. $\left\langle P_{\downarrow}\right\rangle$ values calculated by either procedure fall within the error bars reported in previously published Raman measurements ${ }^{6}$ on a similar doped liquid crystal system and the trend is on the same. The error bars of Fig. 2 are those of the Raman work and are presented to show the overlap. The significance of the low and negative values of $\left\langle P_{4}\right\rangle$ in relation to predictions from mean field theory has been previously discussed. ${ }^{2,8,10}$ We note that the discrepancy with theory cannot be resolved by invoking multiple scattering as our excitation source is of moderate intensity and the fluorescent emission band monitored is well removed from the scattering peak.

Remark: The report ${ }^{11}$ of a similar measurement of $\left\langle P_{2}\right\rangle$ and $\left\langle P_{4}\right\rangle$ in MBBA and the thermotropic liquid crystal EBBA has come to our attention. The authors of this study have assumed an ideal probe molecule with coincident absorption and emission oscillators, suffering no rotational Brownian motion during the lifetime of the excitation. The absence of energy transfer mechanisms was also assumed but not verified. The results of this work for the MBBA liquid crystal system agree closely with that of the previous Raman data cited and our own measurements. Without a proper consideration of and correction for the experimental complications discussed herein, we cannot regard fluorescence depolarization measurements of orientational order as reliable.

\section{APPENDIX}

In a recent publication ${ }^{7}$ we considered the effect of intramolecular energy transfer and diffusional complications on the loss of polarization of radiation emitted from a fluorescent molecule embedded in a uniaxial liquid crystal. Rotational mobility was introduced in simplified form through an isotropic diffusion equation governing the time dependence of an orientational distribution function $g(\theta, t)$ of molecules photoselected in an already ordered environment. The initial photoslected population is described by:

$$
g(\theta, o)=M_{a i}^{2} \cdot f(\theta)
$$

where $M_{a i}^{2}$ is the square of the absorption moment $\left(\cos ^{2} \theta\right.$ for $z$ axis illumination in the geometry considered). Under the assumed symmetry:

$$
g(\theta, t)=\sum_{l \text { eren }} a_{1} \exp [-l(l+1) D t] P_{l}(\cos \theta)
$$

where only diffusion of the long axis of the molecule was considered and $D$ is the appropriate diffusion constant. Time dependent fluorescent intensity elements were then formulated from:

$$
I_{i j}(t)=\iint M_{e j}^{2} g(\theta, t) P(t) \sin \theta d \theta d \phi
$$

where $M_{e j}^{2}$ is the square of the emission moment projected along an axis $j$ and $P(t)=\tau^{-1} e^{-t / \tau}$ is the probability that a molecule emits at time $t$ during the emission lifetime. In the construction of the coefficients of the expansion, Eq. (A2), to satisfy the initial condition, Eq. (A1), $f(\theta)$ was incorrectly factored out. The proper procedure leads to: 


$$
a_{l}=\frac{2 l+1}{2} \cdot \int_{-1}^{1} x^{2} P_{l}(x) d x, \quad x=\cos \theta,
$$

which can be used in the determination of the intensity elements by integration of Eq. (A3). Eq. (1) above is the correct result for the anisotropy of emission. The curves presented in Fig. 3 of the previous article 7 therefore all should approach zero when $\tau / \tau_{R} \gg 1$. The general conclusions of the article otherwise remain valid.

\section{ACKNOWLEDGMENT}

The authors wish to thank Statens teknisk-videnskabelige Forskningsråd for partial support of this work under contract number 516-6569. K-339. We are also grateful to Dr. Donald A. McQuarrie and Dr. Attila Szabo of Indiana University for the correction of the mathematical error discussed in the Appendix.
IIntroduction to Liquid Crystals, edited by E. B. Priestley, P. J. Wojtowicz, and P. Sheng, (Plenum, New York, 1975), p. 72.

${ }^{2}$ G. R. Luckhurst and R. Poupko, Chem. Phys. Lett. 29, 191 (1974).

${ }^{3}$ D. I. Bower, J. Poly. Sci. Poly. Phys. Ed. 10, 2135 (1972).

${ }^{4}$ D. I. Bower, Structure and Properties of Oriented Polymers, edited by I. M. Ward, (Applied Science Publ. Ltd. London, 1975), Chap. 5 .

${ }^{5}$ E. B. Priestley and P. S. Pershan, Mol. Cryst. Liq. Cryst. 23, 369 (1973).

${ }^{6}$ S. Jen, N. A. Clark, P. S. Pershan, and E. B. Priestley, Phys. Rev. Lett. 31, 1552 (1973); J. Chem. Phys. 66, 4635 (1977).

${ }^{7}$ L. L. Chapoy and D. B. DuPre, J. Chem. Phys. 69, 519 (1978).

${ }^{8}$ F. Perrin, J. Phys. 7, 390 (1926).

${ }^{9} \mathrm{G}$. Baur, A. Stieb, and G. Meier, Mol. Cryst. Liq. Cryst. 22, 261 (1973).

${ }^{10}$ G. R. Luckhurst and R. N. Yeates, Mol. Cryst. Liq. Cryst. 34, 57 (1976).

${ }^{11}$ I. Penchev and I. Dozov, Phys. Lett. A 60, 34 (1977). 